\section{$\underset{\substack{\text { hommes } \\ \text { \& migrations }}}{ }$}

\section{Hommes \& migrations}

Revue française de référence sur les dynamiques

migratoires

1299 | 2012

Musulmanes et féministes en Grande-Bretagne

\title{
Une tonne d'acier pour se forger une identité
}

Hélène du Mazaubrun

\section{CpenEdition \\ Journals}

\section{Édition électronique}

URL : http://journals.openedition.org/hommesmigrations/1866

DOI : 10.4000/hommesmigrations.1866

ISSN : 2262-3353

\section{Éditeur}

Musée national de l'histoire de l'immigration

\section{Édition imprimée}

Date de publication : 1 septembre 2012

Pagination : 98-104

ISSN : 1142-852X

Référence électronique

Hélène du Mazaubrun, « Une tonne d'acier pour se forger une identité », Hommes \& migrations [En

ligne], 1299 | 2012, mis en ligne le 29 mai 2013, consulté le 22 septembre 2020. URL : http://

journals.openedition.org/hommesmigrations/1866; DOI : https://doi.org/10.4000/

hommesmigrations.1866

Ce document a été généré automatiquement le 22 septembre 2020.

Tous droits réservés 


\title{
Une tonne d'acier pour se forger une identité
}

\author{
Hélène du Mazaubrun
}

1 En mars 2012, Isabelle Reig contacte la Cité nationale de l'histoire de l'immigration pour faire don d'une fraiseuse ${ }^{1}$ et raconter l'histoire de son père, exilé espagnol. Dans son esprit, c'est une sculpture d'acier, digne des photographies de Willy Ronis. Dans son cœur, elle pèse une tonne. Dans le musée, cette tonne d'acier représente la mémoire de son père qu'elle a voulu "réparer".

2 Emilio Reig est né en 1920 à Torregrosa, dans la province de Lleida, en Espagne. Il a 16 ans lors du coup d'État de la junte militaire de Franco, le 18 juillet 1936. Il s'engage alors, plein d'espoir, dans l'aviation républicaine, parce que ses recruteurs lui promettent de le former, ce qui lui garantirait d'échapper à la mobilisation sur le front. Mais son père ne voit pas les choses sous cet angle et Emilio pour ses 18 ans est envoyé au service militaire de l'armée républicaine. Presque un an plus tard, en janvier 1939, l'armée républicaine est vaincue par la junte militaire. C'est la Retirada ${ }^{2}$. Cet épisode sonne l'exode de milliers de réfugiés espagnols de la guerre civile. À partir de février 1939, ce sont plus de 450000 républicains qui franchissent la frontière suite à la victoire du général Franco et à la chute de la Seconde République espagnole. L'hôpital de Barcelone est évacué. Emilio fait partie des blessés, sa main gauche a explosé avec une grenade. Il part à pied, en plein hiver, et traverse les Pyrénées en direction de la France. Il trouve refuge dans le camp de Septfonds, un des camps les plus importants : 16000 républicains espagnols y sont internés. Le gouvernement Daladier, pressé de décongestionner les autres camps de réfugiés espagnols des Pyrénées-Orientales, avait en effet choisi le Tarn-et-Garonne pour implanter l'un des cinq nouveaux camps destinés à leur hébergement. En vue de rassurer les populations locales, les autorités avaient également affecté plus d'un millier de gardes mobiles, de fantassins, de dragons et des tirailleurs sénégalais. Emilio vit donc ses premiers mois d'exil en compagnie de milliers d'autres réfugiés. La même année, la Seconde Guerre mondiale éclate et le camp de Septfonds se transforme en centre de mobilisation pour les étrangers désireux de s'engager dans les régiments de marche de volontaires étrangers. Mais Emilio refuse 
de rejoindre l'armée française. En août 1942, sous le gouvernement de Vichy, 84 internés juifs appartenant à la $302^{\mathrm{e}}$ compagnie de travailleurs étrangers sont conduits en gare de Caussade, parqués dans un même wagon à destination d'Auschwitz via Drancy. Puis 211 juifs étrangers (hommes, femmes, enfants) sont raflés par la police et la gendarmerie françaises et sont conduits par camion au centre de rassemblement de Septfonds. Dans la nuit du 2 au 3 septembre 1942, les 211 internés sont acheminés en gare de Caussade et intégrés à un important convoi régional qui rejoint Drancy le 4 septembre, pour arriver à Auschwitz cinq jours plus tard. Emilio s'enfuit du camp de Septfonds et traverse à nouveau les Pyrénées, dans l'autre sens, en direction de l'Espagne.De retour dans son village natal, il est attrapé par la garde civile. De 1942 à 1945, il n'a d'autre choix que de servir dans les bataillons disciplinaires de Franco dans le sud de l'Espagne. Il réussit à s'évader, mais il est repris et emprisonné à Lleida. En 1946, la guerre en France est finie et Emilio repasse la frontière pour s'installer à Carcassonne. Il vit à l'hôtel et gagne sa vie en ramassant du chiendent, une herbe qui sert à fabriquer des balais-brosses. Cette période est stoppée par l'arrivée du nylon qui rend cette matière obsolète et Emilio monte à Paris pour trouver un nouveau travail. Il s'est forgé une maxime qu'il a transmise à sa fille : "Plutôt brigand que paysan." Mais rapidement, il se trouve confronté à deux problèmes interdépendants : la recherche d'un emploi et son statut d'exilé. Sa fille résume ainsi la situation : "Sans papiers, pas de travail ; sans travail, pas de papiers." Emilio réclame donc une aide auprès du gouvernement espagnol et des fonds pour une formation lui sont alloués. La France recherche des ouvriers métallurgistes, c'est ainsi qu'il devient fraiseur, alors qu'il est handicapé de la main gauche et que les cours sont donnés en français. Pour sa fille: "Avec la fraiseuse, c'est là que tout a commence ${ }^{3}$."

\section{Grandir à l'ombre des Métallos}

3 En 1949, Emilio rencontre sa femme, Suzanne Manachewitch, âgée de 20 ans. Elle vit seule, dans le salon de coiffure de sa tante, dans une ville limitrophe de Paris, aux Lilas. La grand-mère maternelle d'Isabelle, Marie Kolwsky, est née à Kiev et a immigré en France en 1912. Son grand-père maternel, juif et militant au Parti communiste, est décédé d'une leucémie deux mois avant la déclaration de la guerre. Marie et sa fille, Suzanne, ont échappé aux rafles grâce à un policier qui a refusé de les recenser sur le registre des juifs. Ainsi, elles n'ont jamais porté l'étoile jaune. La mère d'Isabelle est ensuite cachée au sein des jeunesses pétainistes. Âgée de 14 ans, elle sort de la guerre "toujours communiste et juive". Sa tante est déportée en 1942 à Auschwitz, laissant en héritage à sa nièce le salon de coiffure. Bien que la fraiseuse évoque l'histoire paternelle d'Emilio Reig, l'ombre de l'histoire maternelle de Suzanne Manachewitch plane sur la mémoire familiale. En 1949, les parents d'Isabelle se rencontrent à La Java ${ }^{4}$, sur l'air très à la mode de La vie en rose d'Édith Piaf. Emilio s'installe rapidement chez Suzanne, dans le salon de coiffure de 18 mètres carrés. Ils se marient à la mairie du XII ${ }^{\mathrm{e}}$ arrondissement: “C'est ma mère qui a fait toutes les démarches, car elle tenait à avoir une famille normale." Emilio, grâce à sa formation, trouve un emploi qualifié et rémunéré de fraiseur, "en toute légalité", précise Isabelle. Très bricoleur, il utilise son savoir-faire pour retaper une mobylette d'occasion, objet de toute son attention et véhicule de liberté qu'il enfourche pour se rendre à son travail ou partir en voyage de noces sur la Côte d'Azur. Leur fille, Isabelle, naît en 1952. Et, "comble de l'histoire" selon elle, un 18 juillet, date en 1936 du coup d'État de la junte militaire de Franco. Si le 
jour de sa naissance est symbolique, le lieu l'est tout autant. Isabelle Reig naît à la clinique des Métallos, à Paris, rue des Bluets. Les Métallos, c'est aussi la maison de convalescence où Emilio passe quatre mois après une hospitalisation. Les Métallos, c'est également un syndicat qui a acheté une propriété à Baillet et qui offre à ses adhérents un camping où Isabelle et son père ont passé une partie de leurs vacances. Les Métallos, c'est enfin la culture : la Maison des Métallos est un lieu de rencontres qui réunit en particulier des républicains espagnols. Isabelle se rappelle ses dimanches après-midi : "Léo Ferré, Catherine Sauvage, Philippe Clay et Leny Escudero. Tout cela, c'était à la Maison des Métallos; ma curiosité vient d'eux, les Métallos. C'est pour ça que cette fraiseuse représente beaucoup pour mon père, et aussi pour moi." Depuis 1946, Emilio n'est pas retourné en Espagne. En 1954, il est considéré comme "apatride", selon la convention de New York qui s'applique à "toute personne qu'aucun État ne considère comme son ressortissant par application de sa législation ${ }^{5 "}$. Sans patrie, sans nationalité légale, il n'est pas autorisé à passer la frontière. En 1954, il reste donc sur le quai lorsque Isabelle et sa mère partent en Espagne rendre visite à la famille paternelle. Isabelle se souvient de cette séparation et conserve précieusement dans l'album de famille la photographie prise par son père. S'agit-il d'un portrait ? Celui de sa femme et de sa fille à la fenêtre du train? On notera que la focale cadre sous leur visage le panneau de la destination, "Barcelona". Emilio devra patienter encore des années pour que son statut soit modifié. Si des vagues d'amnistie se succèdent, il faut attendre le vote de la loi sur la mémoire historique officiellement appelée "loi pour que soient reconnus et étendus les droits et que soient établis des moyens en faveur de ceux qui ont souffert de persécution ou de violence durant la guerre civile et la dictature". Cette loi espagnole, adoptée en 2007, vise à reconnaître les victimes du franquisme ${ }^{6}$.

\section{Quand une machine-outil devient sert de repère}

4 S'enraciner en France passe par le travail et les papiers, mais aussi par un acte symbolique, celui de poser ses valises dans un logement que l'on fait sien. En 1958, Emilio aspire à l'acquisition d'une maison mais, en raison de son statut d'étranger, il ne peut accéder à un emprunt. Il achète donc comptant un bien délabré, occupé et situé à 300 mètres du camp de Drancy. Pour toutes ces raisons, la famille ne peut y emménager que dix ans plus tard. Pour Isabelle, c'est "l'horreur de Drancy". Vivre à côté d'un camp qui lui rappelle que sa tante a été déportée est aussi lourd à porter que le silence de sa mère sur le sujet. D'ailleurs, Suzanne a abandonné sa culture yiddish, à l'exception de quelques mots et de la cuisine. Isabelle s'interroge aujourd'hui encore sur le "traumatisme" que sa mère a dû subir, ravivé par cette habitation. Son grand-père maternel était un juif russe issu des pogroms et parfaitement intégré, car élu municipal au Blanc-Mesnil ${ }^{7}$. La déportation et la Shoah ont touché directement sa famille. Mais ce n'est que des années plus tard, lorsque les cérémonies à Drancy ont été lancées, que la mère d'Isabelle a commencé à raconter une partie de ses souvenirs à ses petitsenfants.Pour Isabelle, le déracinement paternel et maternel ne peut être conjuré que par une reconnaissance de leur apport à la société française. Le travail de son père et les dimanches riches de rencontres et de culture à la Maison des Métallos deviennent ses racines, à un moment où il fautse construire une identité et une personnalité. La fraiseuse s'impose petit à petit comme un emblème et un repère solide. Cette machine avait été récupérée en 1960, lors de la modernisation de l'entreprise. Rachetée par Emilio, elle avait été installée dans un atelier d'appoint au domicile. Isabelle a alors 8 
ans et elle se souvient du bricolage sur les genoux de son père. Malgré son encombrement, la machine est dans les cartons du déménagement pour Drancy. Pour elle, cet objet est un symbole, celui de l'implication de son père dans la vie française. Emilio est aujourd'hui âgé de 91 ans, à la retraite après trente années dévolues à l'usine Faiveley pour l'équipement ferroviaire. La fraiseuse est toujours entreposée au domicile familial, mais la question de la succession se pose, car pour Isabelle comme pour son père: “Ce n'est pas possible que ça disparaisse."Emilio avait bien envisagé d'en faire don à son beau-fils, puis à son petit-fils, mais il semble que le juste destinataire soit un établissement public. Le besoin de reconnaissance est clairement associé à cette machine et la démarche du don au Musée de l'immigration est emblématique. Cette fraiseuse est bien plus qu'un outil de travail. Bien sûr, elle a servi au forage à l'usine, puis dans l'atelier paternel. Au premier coup d'œil, on en voit l'usage : c'est un objet qui procède au forage par le haut. Sur le plan horizontal, appelé tablier, la pièce à travailler est déposée. Au bras vertical, dans le creux d'un étau, une pièce amovible et spécifique est fixée selon l'opération à traiter, qu'il s'agisse de trouer ou de meuler. Mais la description qu'Isabelle fait de cet objet est révélatrice du regard et de l'affection qu'elle lui porte : "Il faut s'imaginer l'homme, derrière ces machines, qui tournait le tablier et l'étau, se déplaçant latéralement et verticalement. Ce n'était pas automatisé à l'époque. (...) C'est justement à la période de la mécanisation que mon père en récupère une. Grâce à elle, il pouvait faire des choses et se sentir utile. Cette machine représente aussi la fierté d'ouvrier de mon père et son savoir-faire." À vrai dire, pour Isabelle cette fraiseuse est "une véritable sculpture d'acier". La machine est d'ailleurs prise en photo avec les ouvriers dans l'usine. Conservées dans le même album de famille que celui évoqué précédemment, ces images, d'après elle, ressemblent à celles du célèbre photographe Willy Ronis ${ }^{8}$. Cet objet, partie intégrante des meubles et de l'histoire de la famille, est perçu, bien que cela puisse sembler improbable, comme une œuvre d'art.

\section{Donner, reconnaître, réparer}

5 Tandis que la question d'une "filiation" se pose implicitement dans l'esprit d'Emilio, Isabelle se rend à la Maison des Métallos, récemment transformée en espace culturel. "Et là, tout remonte : les concerts de mon enfance, les républicains espagnols... (...) À la Maison des Métallos, il n'y avait que des 'bobos' et là j'ai vu trôner MA fraiseuse dans l'entrée avec les programmes sur le tablier. Tout de suite je l'ai vue, bien briquée ! Bon, il faut dire, je suis bricoleuse, ce n'est pas pour rien d'ailleurs. Je suis très coquette, mais j'aime bien tourner des fraises! (...) Bref, ça a été le flash : la Maison des Métallos et la fraiseuse. En plus, ils avaient gardé le nom! Mais il n'y avait que le nom..." Ainsi, après avoir songé à faire don de la fraiseuse à la Maison des Métallos, Isabelle décide de contacter le Musée national de l'histoire de l'immigration, au Palais de la Porte dorée, en reconnaissance du travail et de l'intégration de son père.Prenant contact avec le musée et racontant l'histoire de son père, Isabelle prend conscience que cette mémoire la concerne elle aussi. La fraiseuse la ramène à sa propre identité : "Nous étions très peu d'enfants de républicains espagnols immigrés à Paris. Comment s'identifier, alors ?" En effet, la plupart des républicains espagnols n'avaient eu d'autre choix que de s'engager dans l'armée française en 1939, et de perdre leur vie au combat. D'autres étaient retournés dans l'Espagne franquiste ou bien s'étaient installés dans le Sud après avoir quitté les camps. Peu de républicains espagnols s'étaient retrouvés à Paris et Isabelle a cherché à comprendre une partie de son histoire auprès de cette 
communauté très restreinte. Ainsi, le don de la fraiseuse, "c'est une reconnaissance, pour moi, de ma nationalité française... Ce serait pour dire: voilà, on a donné une tonne! Une tonne d'acier, c'est à la mesure de tout ce qu'on a apporté à la France, en tant que fils et filles de républicains espagnols". Isabelle se sent porteuse de cette mémoire : "En donnant cette fraiseuse, c'est un peu comme si quelque part je réparais." Finalement, c'est "déjà pour moi que je le fais, peut-être même plus que pour mon père". Le bâtiment du Palais de la Porte Dorée lui était familier, petite, elle était même venue se faire photographier devant ${ }^{9}$. En apprenant que le musée répond favorablement à sa proposition de don, elle décide de s'y rendre pour le visiter, sans prévoir l'émotion qui va l'envahir. "En arrivant au métro Porte Dorée, j'ai commencé à me sentir mal. Lorsque je suis arrivée au musée, je n'arrivais plus à lire. Après, le trou. Les pompiers sont arrivés...je n'ai pas vu grand-chose. Pourtant, je ne suis pas quelqu'un de facilement émotif. Sans doute que cela touche à quelque chose de mal cicatrisé." La visite du musée a ravivé une blessure, celle de la fille d'un immigré espagnol avec son besoin de reconnaissance. Elle se rappelle un épisode de son enfance : “Une fois, j'ai payé une paire de chaussures à mon père. J'avais entendu qu'ile avait besoin de chaussures. Et c'était la fête des Pères. Moi, toute fière, je lui ai offert cette paire. Je me doutais que ça n'irait pas, mais c'était pour l'obliger à ne pas se sacrifier, je pouvais moi aussi me sacrifier, bref réparer un peu. J'avais dix ans". Aujourd'hui, cette blessure n'est pas complètement cicatrisée : “Je crois que je n'ai pas pu réparer. Il ne m'a pas non plus tout transmis, c'était à lui." Finalement, Isabelle comprend grâce au don au musée, que c'est aussi de sa propre histoire qu'il est question. À ses 30 ans, Isabelle a repris des études d'espagnol et entrepris des démarches pour obtenir la double nationalité, française déjà acquise, et espagnole en 2002. Pourtant, son père ne voulait pas que sa fille obtienne le passeport espagnol : "Il n'y aucune raison que tu prennes la nationalité espagnole ; tu n'es pas espagnole." Mais l'appartenance familiale à l'Espagne n'était guère suffisante aux yeux d'Isabelle : "Je ne peux me contenter d'une "identité de cœur". Parce qu'elle ne fréquentait pas la communauté espagnole, il était devenu important pour elle d'obtenir des papiers: "Personne ne sait que je suis espagnole, je n'ai rien pour le prouver." Les lois PasquaDebré ${ }^{10}$ n'avaient pas épargné Isabelle. Elle était née sur le sol français et son père avait obtenu la nationalité française en 1960. Pourtant, elle avait dû prouver sa nationalité auprès de l'État français et renouveler ses papiers. "Depuis que j'ai la double nationalité, ce que je peux dire, c'est que je me sens apaisée." Si ce n'est pour réparer l'histoire de son père ou la sienne, le don de la fraiseuse est perçu tout au moins comme un acte symbolique fort, comme "une humiliation en moins". Une tonne d'acier au musée pour se forger une identité, bâtir une histoire commune et apaiser les mémoires...

\section{NOTES}

1. Une fraiseuse est une machine-outil utilisée pour usiner tous types de pièces mécaniques, à l'unité ou en série, par enlèvement de matière à partir de blocs ou parfois d'ébauches estampées 
ou moulées, à l'aide d'un outil coupant nommé fraise. En dehors de cet outil qui lui a donné son nom, une fraiseuse peut également se voir équipée de foret, de taraud ou d'alésoir.

2. La Retirada, "retraite" en espagnol, désigne l'exode des réfugiés espagnols de la guerre civile.

3. La Retirada, "retraite" en espagnol, désigne l'exode des réfugiés espagnols de la guerre civile.

4. Créée en 1923, cette salle était alors un dancing, parmi les plus réputés de la capitale. La salle a accueilli notamment Django Reinhardt, Jean Gabin, Fréhel et les débuts de Maurice Chevalier et d'Édith Piaf.

5. Convention de New York du 28 septembre 1954 relative au statut des apatrides.

6. Cette loi inclut la reconnaissance de toutes les victimes de la guerre civile espagnole (1936-1939) et de la dictature de Franco (1939-1975), l'ouverture des fosses communes (dans lesquelles se trouvent les restes des victimes) réalisée jusque-là, dans l'attente de subventions de l'État, par des associations privées ou publiques locales, et le retrait des symboles franquistes dans les espaces publics.

7. Le Blanc-Mesnil est une commune française située dans la région Île-de-France, à sept kilomètres des boulevards des Maréchaux et du périphérique au nord-est de Paris, dans le département de la Seine-Saint-Denis.

8. Isabelle Reig trouve que les photographies ressemblent à celles de Willy Ronis dans l'ouvrage Une poétique de l'engagement, publié à l'occasion de l'exposition "Willy Ronis : une poétique de l'engagement" organisée conjointement par le Jeu de Paume et la Monnaie de Paris, présentée à la Monnaie de Paris, du 16 avril au 22 août 2010. En particulier les photographies suivantes: Grève à la Snecma - Kellermann, Paris, 1947 ; Salle de tissage, usine Rhodiaceta, Vaire (Rhône), 1955 ; Le Fil cassé, usine FTB, Haut-Rhin, 1950.

9. Après l'Exposition coloniale, le palais changea d'attribution au gré des évolutions de l'Histoire : Musée permanent des colonies jusqu'en 1935, puis Musée de la France d'outre-mer jusqu'à la fin des années 1950, il accueille en 1961 le Musée des arts africains et océaniens qui deviendra le musée national des arts d'Afrique et d'Océanie en 1990. Avec la création du Musée du quai Branly et la fermeture du MAAO en 2003, seul l'aquarium tropical et les salles historiques du rez-dechaussée restent ouverts au public. Depuis 2007, le bâtiment abrite la Cité nationale de l'histoire de l'immigration.

10. Les lois dites Pasqua-Debré sont trois lois françaises adoptées en 1986, en 1993 et en 1997 dans le but de réguler l'immigration. La première est relative aux conditions d'entrée et de séjour des étrangers en France, dite "loi Pasqua" en référence au ministre de l'Intérieur alors en fonction. La deuxième, dite aussi "loi Pasqua", durcit encore les conditions par rapport à la précédente loi de 1986. La troisième porte sur diverses dispositions relatives à l'immigration, parfois dite "loi Debré", touJours en référence au ministre de l'Intérieur alors en fonction.

\section{AUTEUR}

\section{HÉLÈNE DU MAZAUBRUN}

Chargée des collections, responsable de la Galerie des dons, musée de l'immigration, CNHI. 